\title{
A FUZZY APPROACH TO OPTION PRICING IN A LEVY PROCESS SETTING
}

\author{
PIOTR NOWAK * MACIEJ ROMANIUK *,** \\ * Systems Research Institute \\ Polish Academy of Sciences, ul. Newelska 6, 01-447 Warsaw, Poland \\ e-mail: \{pnowak, mroman\} @ibspan.waw.pl \\ ** The John Paul II Catholic University of Lublin, ul. Konstantynów 1H, 20-708 Lublin, Poland
}

\begin{abstract}
In this paper the problem of European option valuation in a Levy process setting is analysed. In our model the underlying asset follows a geometric Levy process. The jump part of the log-price process, which is a linear combination of Poisson processes, describes upward and downward jumps in price. The proposed pricing method is based on stochastic analysis and the theory of fuzzy sets. We assume that some parameters of the financial instrument cannot be precisely described and therefore they are introduced to the model as fuzzy numbers. Application of fuzzy arithmetic enables us to consider various sources of uncertainty, not only the stochastic one. To obtain the European call option pricing formula we use the minimal entropy martingale measure and Levy characteristics.
\end{abstract}

Keywords: option pricing, Levy processes, minimal entropy martingale measure, fuzzy sets, Monte Carlo simulation.

\section{Introduction}

The Black-Scholes model (Black and Scholes, 1973) is the best known and the most widely used model of option pricing. One of the assumptions made by Black and Scholes is the continuity of the price process. In their approach the underlying asset evolves according to a geometric Brownian motion. The above-mentioned model delivers the completeness of the market and the uniqueness of the derivative pricing. The Black-Scholes formula represents the arbitrage-free option price and it is given in an analytically closed form (see Hull, 1997). However, it is well documented in the finance literature that the Black-Scholes model suffers from two main shortcomings. Firstly, the $(\log )$ returns of the underlying process $S$ in the real market have leptokurtic and skewed to the left distribution (see Davis, 2001). Secondly, the implied volatility is a convex function of the stike price (see Bakshi et al., 1997; Bates, 1996) while, under the Black-Scholes model, this should be a constant function. Such an empirical phenomenon is called the volatility smile.

Many authors have applied discontinuous models to generalize and improve the Black-Scholes model. In Merton's approach the stock price process was modeled as exponential of sum of a Brownian motion with drift and a compound Poisson process with jump sizes following normal distribution (Merton, 1976). Merton solved the option pricing problem assuming that jump risk is not systematic. Kou (2002) obtained European and interest rate options valuation formulas, applying also a jump diffusion model in which the jump sizes have an asymmetric double exponential distribution. Kou and Wang (2004) used the above-mentioned double exponential jump diffusion model for pricing finite horizon American options and path-dependent options. The work of $\mathrm{Xu}$ et al. (2011) was dedicated to foreign equity option pricing.

The authors took into account empirical studies which showed that models with constant volatility do not fully describe equity returns or option prices. They proposed a stochastic volatility model with simultaneous jumps in price and volatility for description of foreign asset prices and exchange rates. Other important approaches, applying pure jump Levy processes to option pricing, are the inverse Gaussian model proposed by Barndorff-Nielsen (1998) as well as the variance gamma model studied by Madan and Seneta (1990).

One of the purposes of this paper is to improve the pricing model. Like in the above mentioned approaches, we take into account the fact that in the real market asset prices do jump, and some risks cannot be handled within continuous-path models. We consider the independent 
sum of a Brownian motion with drift and a linear combination of a finite number of independent Poisson processes as the model of the log-price $Y$. The so defined process $Y$ is a Levy process and its discontinuous part can model positive and negative jumps of the underlying asset caused by external shocks. We continue and generalize our previous approach (Nowak and Romaniuk, 2010). The proposed log-price process is an extension of the previous model considered with one possible Poisson component. A similar model for two Poisson components was considered by Nowak (2011) in a preliminary version, without Monte Carlo simulations. In the paper, stochastic analysis and fuzzy sets theory are employed to prove the option pricing formula.

An approach similar to ours was applied to the Black-Scholes model by Wu (2004) along with its improved version (Wu, 2007). Yoshida (2003) also considered the Black-Scholes model in a fuzzy environment. However, rational expected option price proposed by him depends on a fuzzy goal, which represents the buyer's/writer's subjective judgement and therefore can be different for different financial analysts. Zhang et al. (2012) obtained the European option fuzzy pricing formula based on Kou's double exponential jump diffusion model. They also proposed a crisp possibilistic mean option pricing formula by using the possibilistic mean values of fuzzy numbers. Fuzzy numbers may be also used in other areas of financial mathematics, e.g., financial time series forecasting (see $\mathrm{Li}$ and Chiang, 2012).

Within the stochastic analysis used in our discussion, martingale theory and its application play important roles in this paper. We also use characteristics of the Levy process (see Shiryaev, 1999) to find the valuation expression. As the equivalent martingale measure we apply the Minimal Entropy Martingale Measure (MEMM). This measure is used by many authors for option pricing (see El Karoui and Rouge, 2000; Frittelli, 2000). The relation of the MEMM to market efficiency and maximisation of exponential utility makes the measure economically meaningful (see Ssebugenyi, 2011). As we have mentioned above, we use fuzzy set theory and fuzzy arithmetic (see Zadeh, 1965). A fluctuating financial market and the lack of detailed information cause that many parameters of the model cannot always be described in a precise sense. We take into account this type of uncertainty. Experts' opinions or imprecise estimates are introduced to the model in the form of fuzzy numbers. A financial analyst, using fuzzy parameters in the valuation formula, can obtain a tool to pick the option price with an acceptable membership degree for later use (see $\mathrm{Wu}, 2004$ ).

Computation techniques, which illustrate the theoretical results, will be mainly applied to the European call options. However, the proposed pricing method can also be used for other derivatives.

The paper is organized as follows. Section 2 contains preliminaries from the fuzzy arithmetic. In Section 3 we present basic definitions and facts concerning stochastic analysis and the minimal entropy martingale measure. In Section 4 we propose a geometric Levy process as the model of the underlying asset and we prove the European call option pricing formula. Section 5 is devoted to evaluation of the derivative with fuzzy parameters. In Section 6 we conduct Monte Carlo simulations to illustrate and analyse the previously obtained valuation expression. The last section contains concluding remarks.

\section{Fuzzy sets: Notation and definitions}

Let $\tilde{A}$ be a fuzzy subset of the set of real numbers $\mathbb{R}$. We denote by $\mu_{\tilde{A}}$ its membership function $\mu_{\tilde{A}}: \mathbb{R} \rightarrow[0,1]$, and by $\tilde{A}_{\alpha}=\left\{x: \mu_{\tilde{A}} \geq \alpha\right\}$ the $\alpha$-level set of $\tilde{A}$, where $\tilde{A}_{0}$ is the closure of the set $\left\{x: \mu_{\tilde{A}} \neq 0\right\}$.

Let $\tilde{a}$ be a fuzzy number. We assume that $\mu_{\tilde{a}}$ is an upper semicontinuous function. Then the $\alpha$-level set $\tilde{a}_{\alpha}$ is a closed interval of the form $\tilde{a}_{\alpha}=\left[\tilde{a}_{\alpha}^{L}, \tilde{a}_{\alpha}^{U}\right]$ (see, e.g. Zadeh, 1965).

Next we recall the arithmetic of fuzzy numbers. Let $\oplus, \ominus, \otimes$ and $\oslash$ be binary operators between fuzzy numbers, and let,,$+- \times$ and / be standard operators between crisp real numbers. Let $\odot$ be an operator from the first group and let $\circ$ be the operator from the second group corresponding to $\odot$. Then, for fuzzy numbers $\tilde{a}$ and $\tilde{b}$, the membership function of $\tilde{a} \odot \tilde{b}$ is defined by (see Zhou, 2002)

$$
\mu_{\tilde{a} \odot \tilde{b}}(z)=\sup _{(x, y): x \circ y=z} \min \left\{\mu_{\tilde{a}}(x), \mu_{\tilde{b}}(y)\right\} .
$$

Let $\odot_{\text {int }}$ be a binary operator $\oplus_{\text {int }}, \ominus_{\text {int }}, \otimes_{\text {int }}$ or $\oslash_{\text {int }}$ between two closed intervals $[a, b]$ and $[c, d]$. Then

$$
\begin{aligned}
{[a, b] \odot \text { int }[c, d]=\{z} & \in \mathbb{R}: z=x \circ y, \\
& \forall x \in[a, b], \quad \forall y \in[c, d]\},
\end{aligned}
$$

where $\circ$ is the standard crisp operator corresponding to $\odot_{\text {int }}$ (i.e.,,,$+- \times$ or $/$, if the interval $[c, d]$ does not contain zero in the last case).

Therefore, if $\tilde{a}, \tilde{b}$ are fuzzy numbers, then $\tilde{a} \odot \tilde{b}$ is also a fuzzy number defined via its $\alpha$-level sets by

$$
\begin{gathered}
(\tilde{a} \oplus \tilde{b})_{\alpha}=\tilde{a}_{\alpha} \oplus_{\text {int }} \tilde{b}_{\alpha}=\left[\tilde{a}_{\alpha}^{L}+\tilde{b}_{\alpha}^{L}, \tilde{a}_{\alpha}^{U}+\tilde{b}_{\alpha}^{U}\right], \\
(\tilde{a} \ominus \tilde{b})_{\alpha}=\tilde{a}_{\alpha} \ominus_{\text {int }} \tilde{b}_{\alpha}=\left[\tilde{a}_{\alpha}^{L}-\tilde{b}_{\alpha}^{U}, \tilde{a}_{\alpha}^{U}-\tilde{b}_{\alpha}^{L}\right], \\
(\tilde{a} \otimes \tilde{b})_{\alpha} \\
=\tilde{a}_{\alpha} \otimes_{\operatorname{int}} \tilde{b}_{\alpha} \\
=\left[\min \left\{\tilde{a}_{\alpha}^{L} \tilde{b}_{\alpha}^{L}, \tilde{a}_{\alpha}^{L} \tilde{b}_{\alpha}^{U}, \tilde{a}_{\alpha}^{U} \tilde{b}_{\alpha}^{L}, \tilde{a}_{\alpha}^{U} \tilde{b}_{\alpha}^{U}\right\},\right. \\
\left.\quad \max \left\{\tilde{a}_{\alpha}^{L} \tilde{b}_{\alpha}^{L}, \tilde{a}_{\alpha}^{L} \tilde{b}_{\alpha}^{U}, \tilde{a}_{\alpha}^{U} \tilde{b}_{\alpha}^{L}, \tilde{a}_{\alpha}^{U} \tilde{b}_{\alpha}^{U}\right\}\right],
\end{gathered}
$$




$$
\begin{aligned}
(\tilde{a} \oslash \tilde{b})_{\alpha} & \\
= & \tilde{a}_{\alpha} \oslash_{\operatorname{int}} \tilde{b}_{\alpha} \\
= & {\left[\min \left\{\tilde{a}_{\alpha}^{L} / \tilde{b}_{\alpha}^{L}, \tilde{a}_{\alpha}^{L} / \tilde{b}_{\alpha}^{U}, \tilde{a}_{\alpha}^{U} / \tilde{b}_{\alpha}^{L}, \tilde{a}_{\alpha}^{U} / \tilde{b}_{\alpha}^{U}\right\},\right.} \\
& \left.\max \left\{\tilde{a}_{\alpha}^{L} / \tilde{b}_{\alpha}^{L}, \tilde{a}_{\alpha}^{L} / \tilde{b}_{\alpha}^{U}, \tilde{a}_{\alpha}^{U} / \tilde{b}_{\alpha}^{L}, \tilde{a}_{\alpha}^{U} / \tilde{b}_{\alpha}^{U}\right\}\right],
\end{aligned}
$$

if the $\alpha$-level set $\tilde{b}_{\alpha}$ does not contain zero for all $\alpha \in[0,1]$ in the case of $\oslash$.

Let $\mathcal{F}(\mathbb{R})$ be the set of all fuzzy numbers.

Proposition 1. Let $f: \mathbb{R} \rightarrow \mathbb{R}$ be a function and let $\{x \in$ $\mathbb{R}: f(x)=y\}$ be a compact set for each $y \in \mathbb{R}$. Then $f$ induces a fuzzy-valued function $\tilde{f}: \mathcal{F}(\mathbb{R}) \rightarrow \mathcal{F}(\mathbb{R})$ via the extension principle and for each $\tilde{\Lambda} \in \mathcal{F}(\mathbb{R})$ the $\alpha$ level set of $\tilde{f}(\tilde{\Lambda})$ has the form $\tilde{f}(\tilde{\Lambda})_{\alpha}=\left\{f(x): x \in \tilde{\Lambda}_{\alpha}\right\}$.

The proof of this proposition may be found in the work of Wu (2004).

Now we introduce definitions of some special cases of fuzzy numbers which may be useful in our discussion.

A triangular fuzzy number $\tilde{a}$ is a fuzzy number with the membership function defined by

$$
\mu_{\tilde{a}}(x)= \begin{cases}\frac{x-a_{1}}{a_{2}-a_{1}} & \text { for } a_{1} \leq x \leq a_{2}, \\ \frac{x-a_{3}}{a_{2}-a_{3}} & \text { for } a_{2} \leq x \leq a_{3}, \\ 0 & \text { otherwise }\end{cases}
$$

where $\left[a_{1}, a_{3}\right]$ is the supporting interval and the membership function has peak in $a_{2}$. The triangular fuzzy number $\tilde{a}$ is denoted further by

$$
\tilde{a}=\left(a_{1}, a_{2}, a_{3}\right) .
$$

Left-Right (L-R) fuzzy numbers are generalizations of triangular fuzzy numbers (see, e.g., Bardossy and Duckstein, 1995; Dubois and Prade, 1980), where the linear functions used in (3) are replaced by monotonic functions. We apply L-R numbers in our numerical analysis of the fuzzy pricing formula for the European call option (see Section 6).

Definition 1. A fuzzy number $\tilde{a}$ is called an L-R number if its membership function has the form

$$
\mu_{\tilde{a}}(x)= \begin{cases}L\left(\frac{a_{2}-x}{a_{2}-a_{1}}\right) & \text { for } a_{1} \leq x \leq a_{2}, \\ R\left(\frac{x-a_{2}}{a_{3}-a_{2}}\right) & \text { for } a_{2} \leq x \leq a_{3}, \\ 0 & \text { otherwise, }\end{cases}
$$

where $L$ and $R$ are continuous strictly decreasing functions defined on $[0,1]$ with values in $[0,1]$ satisfying the following conditions:

$$
L(x)=R(x)= \begin{cases}1 & \text { if } x=0 \\ 0 & \text { if } x=1\end{cases}
$$

The L-R fuzzy number $\tilde{a}$ is denoted further by

$$
\tilde{a}=\left(a_{1}, a_{2}, a_{3}\right)_{L R} .
$$

\section{MEMM for Levy processes}

We recall some basic facts about the stochastic analysis and the martingale method.

Let $\left(\Omega, \mathcal{F},\left(\mathcal{F}_{t}\right)_{t \in[0, T]}, P\right)$ be a probability space with filtration satisfying standard assumptions. Let $T<$ $\infty$ be a time horizon.

A stochastic process $H=\left(H_{t}\right)_{t \in[0, T]}$ is cadlag if its trajectories are functions which are right-continuous with left limits.

$H$ is $\left(\mathcal{F}_{t}\right)$-adapted if $H_{t}$ is $\left(\mathcal{F}_{t}\right)$-measurable for each $t \in[0, T]$.

A probability measure $Q$ on $(\Omega, \mathcal{F})$ is absolutely continuous with respect to $P(Q<<P)$ if, for all $A \in \mathcal{F}$,

$$
P(A)=0 \Rightarrow Q(A)=0
$$

and it is equivalent to $P$ if $P$ and $Q$ have the same sets with zero measure.

Let $\mathcal{S}_{t}$ be an $\left(\mathcal{F}_{t}\right)$-adapted cadlag stochastic process describing the underlying asset. Let $r$ denote a constant risk-free interest rate, and let

$$
\mathcal{Z}_{t}=e^{-r t} \mathcal{S}_{t}
$$

be the discounted process of values of the underlying asset. We have to find a measure $Q$ equivalent to $P$ for which $\mathcal{Z}_{t}$ is a martingale.

The next step is to find the form of the process $\mathcal{S}_{t}$ with respect to this new probability measure $Q$. The price of a derivative with a payment function $f$ is given by the formula

$$
C_{t}=\exp (-r(T-t)) \mathbb{E}^{Q}\left(f(S) \mid \mathcal{F}_{t}\right), \quad t \in[0, T] .
$$

A cadlag stochastically continuous process $Y=$ $\left(Y_{t}\right)_{t \in[0, T]}, Y_{0}=0$ a.s., is called a Levy process if it satisfies the following conditions:

1. $Y_{t}-Y_{s}$ is independent of $\mathcal{F}_{s}$ for all $0 \leq s \leq t \leq T$.

2. $Y_{t}-Y_{s}$ and $Y_{t-s}$ have the same distributions for all $0 \leq s \leq t \leq T$.

We assume that a truncation function $h$ is defined by the formula $h(x)=x I_{|x| \leq 1}$. We denote by $\mathcal{M}(\mathbb{R})$ the space of non-negative measures on $\mathbb{R}$. For a Levy process $Y$ local characteristics $\left(B_{t}, C_{t}, \nu_{t}\right)$ are defined by the Levy-Khintchine formula

$$
\begin{aligned}
\varphi_{t}(\theta)= & \mathbb{E}^{P} e^{i \theta Y_{t}} \\
= & \exp \left\{i \theta B_{t}-\frac{1}{2} \theta^{2} C_{t}\right. \\
& \left.+\int_{\mathbb{R}}\left(e^{i \theta x}-1-i \theta h(x)\right) \nu_{t}(\mathrm{~d} x)\right\},
\end{aligned}
$$


where $\varphi_{t}(\theta)$ is the characteristic function of $Y_{t}$,

$$
\begin{gathered}
B_{t}:[0, T] \rightarrow \mathbb{R}, \quad B_{t}=b t, \\
C_{t}:[0, T] \rightarrow \mathbb{R}, \quad C_{t}=c t, \\
\nu_{t}:[0, T] \rightarrow \mathcal{M}(\mathbb{R}), \quad \nu_{t}(\mathrm{~d} x)=\nu(\mathrm{d} x) t, \\
\nu(\{0\})=0, \quad \int_{\mathbb{R}}\left(|x|^{2} \wedge 1\right) \nu(\mathrm{d} x)<\infty,
\end{gathered}
$$

$b, c \in \mathbb{R}$ and $\nu \in \mathcal{M}(\mathbb{R})$. Moreover, only constant $b$ depends on the form of $h$. For details concerning characteristics of Levy processes and their generalizations for semimartingales we refer the reader to the works of Shiryaev (1999) as well as Jacod and Shiryaev (1987).

A stochastic process $S=\left(S_{t}\right)_{t \in[0, T]}$ is called a geometric Levy process if it can be written in the following form:

$$
S_{t}=S_{0} \exp \left(Y_{t}\right), \quad t \in[0, T],
$$

where $Y_{t}$ is a Levy process.

Throughout this paper we assume that $S$ is of the form (7), $\mathcal{F}=\mathcal{F}_{T}$ and for $t \in[0, T]$

$$
\mathcal{F}_{t}=\sigma\left(S_{s}, s \in[0, t]\right)=\sigma\left(Y_{s}, s \in[0, t]\right) .
$$

The relative entropy $I(Q, P)$ of $Q$ with respect to $P$ is defined by

$$
I(Q, P)= \begin{cases}E_{P}\left(\frac{\mathrm{d} Q}{\mathrm{~d} P} \ln \left(\frac{\mathrm{d} Q}{\mathrm{~d} P}\right)\right) & \text { if } Q \ll P, \\ +\infty & \text { otherwise }\end{cases}
$$

If an equivalent martingale measure $P_{0}$ satisfies the inequality

$$
I\left(P_{0}, P\right) \leq I(Q, P)
$$

for all equivalent martingale measures $Q$, then $P_{0}$ is called the minimal entropy martingale measure.

Let

$$
\begin{aligned}
& g^{(\text {MEMM })}(\theta) \\
& =b+\left(\frac{1}{2}+\theta\right) c \\
& \quad+\int_{\{|x| \leq 1\}}\left(\left(e^{x}-1\right) e^{\theta\left(e^{x}-1\right)}-x\right) \nu(\mathrm{d} x) \\
& \quad+\int_{\{|x|>1\}}\left(e^{x}-1\right) e^{\theta\left(e^{x}-1\right)} \nu(\mathrm{d} x) .
\end{aligned}
$$

The following theorem of Miyahara (2004), proved by Fujiwara and Miyahara (2003), will be useful in our further discussion.

Theorem 1. If the equation

$$
g^{(\mathrm{MEMM})}(\theta)=r
$$

has a solution $\theta_{0}$, then the minimal entropy martingale measure $P_{0}$ of $S$ exists. The process $Y$ is also a Levy process under $P_{0}$ and the generating triplet $\left(b_{0}, c_{0}, \nu_{0}\right)$ of $Y$ under $P_{0}$ has the form

$$
\begin{gathered}
b_{0}=b+\theta_{0} c+\int_{\{|x| \leq 1\}} x\left(e^{\theta_{0}\left(e^{x}-1\right)}-1\right) \nu(\mathrm{d} x), \\
c_{0}=c, \\
\nu_{0}(\mathrm{~d} x)=e^{\theta_{0}\left(e^{x}-1\right)} \nu(\mathrm{d} x) .
\end{gathered}
$$

\section{Pricing with crisp parameters}

Let $\left(\Omega, \mathcal{F},\left(\mathcal{F}_{t}\right)_{t \in[0, T]}, P\right)$ be a probability space with filtration defined in the previous section. Let $T<\infty$.

We assume that the price of the underlying asset $S_{t}$ is the geometric Levy process given by (7), where

$$
Y_{t}=\mu t+\sigma W_{t}+k_{1} N_{t}^{\kappa_{1}}+k_{2} N_{t}^{\kappa_{2}}+\cdots+k_{D} N_{t}^{\kappa_{D}},
$$

$W_{t}$ is a Brownian motion, $D$ is a positive integer, $\sigma>0, \mu, k_{1}, k_{2}, \ldots, k_{D} \in \mathbb{R}, N_{t}^{\kappa_{1}}, N_{t}^{\kappa_{2}}, \ldots, N_{t}^{\kappa_{D}}$ are independent Poisson processes with the intensities $\kappa_{1}>0, \kappa_{2}>0, \ldots, \kappa_{D}>0$. We also assume that $W_{t}$ and the Poisson processes are independent. The numbers $k_{1}, k_{2}, \ldots, k_{D}$ describe the heights of jumps in the underlying asset price.

The process (9) can be also described as the compound Poisson process of the form

$$
Y_{t}=\mu t+\sigma W_{t}+\sum_{i=1}^{N_{t}^{\kappa}} \xi_{i}
$$

where $W_{t}$ is also a Brownian motion, $\sigma>0, \mu, k \in \mathbb{R}, N_{t}^{\kappa}$ is a Poisson process, independent of $W_{t}$, with the intensity $\kappa=\kappa_{1}+\kappa_{2}+\cdots+\kappa_{D}$, and independent random variables $\left\{\xi_{i}\right\}_{i=1,2, \ldots}$ take value $k_{j}$ with probability $p_{j}=\kappa_{j} / \kappa$ $, j=1,2, \ldots, D$. From a probabilistic point of view they can be treated as the same processes, since they have the same finite dimensional distributions. In particular, they have the same characteristic triplets. The equivalence of similiar type between a GPL process, which is a linear combination of independent Poisson processes with positive integer coefficients, and a corresponding compound Poisson process was discussed and explained by Brigo et al. (2007). In comparison with (10), the form (9) is more convenient to obtain the pricing formula for the European call option.

Theorem 2. The price of the European call option with the strike price $K$ and the payment function $f(x)=(x-$ 
$K)_{+}$at time 0 is given by

$$
\begin{aligned}
& C_{0} \\
& =e^{-\left(\kappa_{1}^{\prime}+\kappa_{2}^{\prime}+\cdots+\kappa_{D}^{\prime}\right) T} \\
& \quad \times \sum_{m_{1}=0}^{\infty} \sum_{m_{2}=0}^{\infty} \cdots \sum_{m_{D}=0}^{\infty} \frac{\left(\kappa_{1}^{\prime}\right)^{m_{1}}}{m_{1} !} \frac{\left(\kappa_{2}^{\prime}\right)^{m_{2}}}{m_{2} !} \cdots \frac{\left(\kappa_{D}^{\prime}\right)^{m_{D}}}{m_{D} !} \\
& \quad \times T^{m_{1}+m_{2}+\cdots+m_{D}} I_{m_{1}, m_{2}, \ldots, m_{D}},
\end{aligned}
$$

where

$$
\begin{aligned}
& I_{m_{1}, m_{2}, \ldots, m_{D}} \\
& =S_{0} e^{\left(\mu_{1}-r\right) T+\frac{\sigma^{2} T}{2}+k^{\mathbf{m}}} \Phi\left(d_{+}^{\mathbf{m}}\right)-e^{-r T} K \Phi\left(d_{-}^{\mathbf{m}}\right),
\end{aligned}
$$

$\Phi$ is the cumulative distribution function of the standard normal distribution and $\theta_{0}$ is the solution of the equation

$$
\begin{gathered}
\mu+\left(\frac{1}{2}+\theta\right) \sigma^{2}+\sum_{i=1}^{D} \kappa_{i}\left(e^{k_{i}}-1\right) e^{\theta\left(e^{k_{i}}-1\right)}=r,(11) \\
k^{\mathbf{m}}=k_{1} m_{1}+k_{2} m_{2}+\cdots+k_{D} m_{D}, \\
\mu_{1}=\mu+\theta_{0} \sigma^{2}, \quad \kappa_{i}^{\prime}=\kappa_{i} e^{\theta_{0}\left(e^{k_{i}}-1\right)}, \quad i=1,2, \ldots, D, \\
d_{-}^{\mathbf{m}}=\frac{\ln \frac{S_{0}}{K}+\mu_{1} T+k^{\mathbf{m}}}{\sigma \sqrt{T}}, \\
d_{+}^{\mathbf{m}}=\frac{\ln \frac{S_{0}}{K}+\mu_{1} T+\sigma^{2} T+k^{\mathbf{m}}}{\sigma \sqrt{T}} .
\end{gathered}
$$

Proof. We apply Theorem 1 to price the option. Equation (8) has the form (11). Since $g$ is an increasing continuous function of $\theta \lim _{\theta \rightarrow-\infty} g(\theta)=-\infty$ and $\lim _{\theta \rightarrow \infty} g(\theta)=$ $\infty$, the above equation has a unique solution. We denote it by $\theta_{0}$. According to Theorem 1 , with respect to $P_{0}, Y$ has the form

$$
\begin{aligned}
Y_{t}= & \left(\mu+\theta_{0} \sigma^{2}\right) t+\sigma W_{t}^{0}+k_{1} N_{t}^{\kappa_{1}^{\prime}}+k_{2} N_{t}^{\kappa_{2}^{\prime}} \\
& +\cdots+k_{D} N_{t}^{\kappa_{D}^{\prime}}, \quad t \in[0, T]
\end{aligned}
$$

where $W^{0}$ is a Brownian motion, $N_{t}^{\kappa_{1}^{\prime}}, N_{t}^{\kappa_{2}^{\prime}}, \ldots, N_{t}^{\kappa_{D}^{\prime}}$ are Poisson processes with respect to $P_{0}$, and all these processes are independent.
The price of the derivative is given by

$$
\begin{aligned}
& C_{0}=e^{-r T} \mathbb{E}^{P_{0}}\left(S_{T}-K\right)^{+} \\
& =e^{-r T} \mathbb{E}^{P_{0}}\left(S_{T}-K\right) I_{\left\{S_{T}>K\right\}}
\end{aligned}
$$

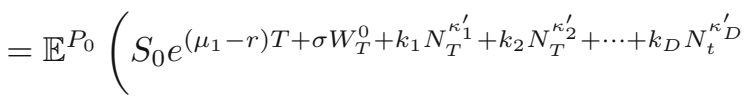

$$
\begin{aligned}
& \left.-e^{-r T} K\right) I_{\left\{S_{T}>K\right\}} \\
& =\mathbb{E}^{P_{0}} \sum_{m_{1}=0}^{\infty} \sum_{m_{2}=0}^{\infty} \cdots \\
& \sum_{m_{D}=0}^{\infty} I_{\left\{N_{T}^{\kappa_{1}^{\prime}}=m_{1}, N_{T}^{\kappa_{2}^{\prime}}=m_{2}, \ldots, N_{T}^{\kappa_{D}^{\prime}}=m_{D}\right\}} \\
& \times\left(S_{0} e^{\left(\mu_{1}-r\right) T+\sigma W_{T}^{0}+k^{\mathbf{m}}}-e^{-r T} K\right) \\
& \times I_{\left\{\left(\mu_{1}-r\right) T+\sigma W_{T}^{0}+k^{\mathbf{m}}>\ln \frac{K}{S_{0}}-r T\right\}} \\
& =e^{-\left(\kappa_{1}^{\prime}+\kappa_{2}^{\prime}+\cdots+\kappa_{D}^{\prime}\right) T} \\
& \times \sum_{m_{1}=0}^{\infty} \sum_{m_{2}=0}^{\infty} \ldots \sum_{m_{D}=0}^{\infty} \frac{\left(\kappa_{1}^{\prime}\right)^{m_{1}}}{m_{1} !} \frac{\left(\kappa_{2}^{\prime}\right)^{m_{2}}}{m_{2} !} \ldots \frac{\left(\kappa_{D}^{\prime}\right)^{m_{D}}}{m_{D} !} \\
& \times T^{m_{1}+m_{2}+\cdots+m_{D}} \text {. } \\
& \times \mathbb{E}^{P_{0}}\left(S_{0} e^{\left(\mu_{1}-r\right) T+\sigma W_{T}^{0}+k^{\mathbf{m}}}-e^{-r T} K\right) \\
& \times I_{\left\{\left(\mu_{1}-r\right) T+\sigma W_{T}^{0}+k^{\mathbf{m}}>\ln \frac{K}{S_{0}}-r T\right\}} .
\end{aligned}
$$

Since

$$
\left(\mu_{1}-r\right) T+\sigma W_{T}^{0}+k^{\mathbf{m}} \sim N\left(\left(\mu_{1}-r\right) T+k^{\mathbf{m}}, \sigma \sqrt{T}\right),
$$

applying standard integral operations we obtain

$$
\begin{aligned}
& \mathbb{E}^{P_{0}}\left(S_{0} e^{\left(\mu_{1}-r\right) T+\sigma W_{T}^{0}+k^{\mathbf{m}}}-e^{-r T} K\right) \\
& \times I_{\left\{\left(\mu_{1}-r\right) T+\sigma W_{T}^{0}+k^{\mathbf{m}}>\ln \frac{K}{S_{0}}-r T\right\}} \\
& =S_{0} e^{\left(\mu_{1}-r\right) T+\frac{\sigma^{2} T}{2}+k^{\mathbf{m}}} \Phi\left(d_{+}^{\mathbf{m}}\right)-e^{-r T} K \Phi\left(d_{-}^{\mathbf{m}}\right) \\
& =I_{m_{1}, m_{2}, \ldots, m_{D}} .
\end{aligned}
$$

Applying the Taylor expansion of the function exp, in a further part of the paper, we shall replace the pricing formula by

$$
\begin{aligned}
C_{0}= & e^{-\left(\kappa_{1}^{\prime}+\kappa_{2}^{\prime}+\cdots+\kappa_{D}^{\prime}\right) T} \\
& \times \sum_{m_{1}=0}^{N} \sum_{m_{2}=0}^{N} \cdots \sum_{m_{D}=0}^{N} \frac{\left(\kappa_{1}^{\prime}\right)^{m_{1}}}{m_{1} !} \frac{\left(\kappa_{2}^{\prime}\right)^{m_{2}}}{m_{2} !} \cdots \frac{\left(\kappa_{D}^{\prime}\right)^{m_{D}}}{m_{D} !} \\
& \times T^{m_{1}+m_{2}+\cdots+m_{D}} I_{m_{1}, m_{2}, \ldots, m_{D}}
\end{aligned}
$$

for a sufficiently large $N$. 


\section{Pricing with fuzzy parameters}

In this section we will take into account possible errors and uncertainties which arise from the estimation of financial market parameters. We introduce fuzzy parameters, assuming that they are L-R numbers. To indicate their fuzziness, we will write the symbol $\sim$ on top of them. Other parameters will be treated as crisp numbers. The methodology similar to the one applied below can be found in the work of Wu (2004).

Let us assume that the drift $\mu$, the volatility $\sigma$, the interest rate $r, k_{1}, k_{2}, \ldots, k_{D}, \kappa_{1}, \kappa_{2}, \ldots, \kappa_{D}$ are not known precisely. Therefore we will treat them as L-R fuzzy numbers $\tilde{\mu}, \tilde{\sigma}, \tilde{r}, \tilde{k}_{1}, \tilde{k}_{2}, \ldots, \tilde{k}_{D}, \tilde{\kappa}_{1}, \tilde{\kappa}_{2}, \ldots, \tilde{\kappa}_{D}$. Let $\mu^{*}, \sigma^{*}, r^{*}, k_{1}^{*}, k_{2}^{*}, \ldots, k_{D}^{*}, \kappa_{1}^{*}, \kappa_{2}^{*}, \ldots, \kappa_{D}^{*}$ be their defuzzified versions. We obtain the following form of the pricing formula (13):

$$
\begin{aligned}
\tilde{C}_{0}= & e^{-\left(\tilde{\kappa}_{1}^{\prime} \oplus \tilde{\kappa}_{2}^{\prime} \oplus \cdots \oplus \tilde{\kappa}_{D}^{\prime}\right) \otimes T} \\
& \otimes \bigoplus_{m_{1}=0}^{N} \bigoplus_{m_{2}=0}^{N} \cdots \bigoplus_{m_{D}=0}^{N}\left(\left(\tilde{\kappa}_{1}^{\prime}\right)^{m_{1}} \oslash m_{1} !\right) \\
& \otimes\left(\left(\tilde{\kappa}_{2}^{\prime}\right)^{m_{2}} \oslash m_{2} !\right) \\
& \otimes \ldots \otimes\left(\left(\tilde{\kappa}_{D}^{\prime}\right)^{m_{D}} \oslash m_{D} !\right) \otimes T^{m_{1}+m_{2}+\cdots+m_{D}} \\
& \otimes\left(S_{0} \otimes e^{\left[\left(\left(\tilde{\mu}^{\prime} \ominus \tilde{r}\right) \otimes T\right) \oplus(\tilde{\sigma} \otimes \tilde{\sigma} \otimes T \oslash 2) \oplus \tilde{k}^{\mathbf{m}}\right]}\right. \\
& \left.\otimes \tilde{\Phi}\left(\tilde{d}_{+}^{\mathbf{m}}\right) \ominus\left(e^{-\tilde{r} \otimes T} \otimes K \otimes \tilde{\Phi}\left(\tilde{d}_{-}^{\mathbf{m}}\right)\right)\right),
\end{aligned}
$$

where

$$
\begin{aligned}
\tilde{k}^{\mathbf{m}}= & \left(\tilde{k}_{1} \otimes m_{1}\right) \oplus\left(\tilde{k}_{2} \otimes m_{2}\right) \oplus \cdots \oplus\left(\tilde{k}_{D} \otimes m_{D}\right), \\
\tilde{\mu}^{\prime}=\tilde{\mu} \oplus\left(\theta_{0} \otimes \tilde{\sigma} \otimes \tilde{\sigma}\right), & \tilde{\kappa}_{i}^{\prime}=\tilde{\kappa}_{i} \otimes e^{\theta_{0} \otimes\left(e^{\tilde{k}_{i}} \ominus 1\right)}, \quad i=1,2, \ldots, D, \\
\tilde{d}_{-}^{\mathbf{m}}= & {\left[\ln \left(S_{0} \oslash K\right) \oplus\left(\tilde{\mu}^{\prime} \otimes T\right) \oplus \tilde{k}^{\mathbf{m}}\right] \oslash(\tilde{\sigma} \otimes \sqrt{T}), } \\
\tilde{d}_{+}^{\mathbf{m}}= & {\left[\ln \left(S_{0} \oslash K\right) \oplus\left(\tilde{\mu}^{\prime} \otimes T\right) \oplus(\tilde{\sigma} \otimes \tilde{\sigma} \otimes T) \oplus \tilde{k}^{\mathbf{m}}\right] } \\
& \oslash(\tilde{\sigma} \otimes \sqrt{T}),
\end{aligned}
$$

and $\theta_{0}$ is the solution (with respect to $\theta$ ) of the equation

$\mu^{*}+\left(\sigma^{*}\right)^{2}\left(\frac{1}{2}+\theta\right)+\sum_{i=1}^{D} \kappa_{i}^{*} e^{\theta\left(e^{k_{i}^{*}}-1\right)}\left(e^{k_{i}^{*}}-1\right)=r^{*}$

In Eqn. 15 the parameters with $*$ are defuzzified using one of the maximum methods (e.g., the mean of maximum method) for L-R numbers. The existence of the solution of (15) follows from the same argument as in the proof of Theorem 2. set of

Applying Proposition 1 we can calculate the $\alpha$-level

$$
\left(\tilde{C}_{0}\right)_{\alpha}=\left[\tilde{C}_{0 \alpha}^{L}, \tilde{C}_{0 \alpha}^{U}\right]
$$

using corresponding combinations of $\tilde{r}_{\alpha}^{L}$ or $\tilde{r}_{\alpha}^{U}, \tilde{\mu}_{\alpha}^{L}$ or $\tilde{\mu}_{\alpha}^{U}, \tilde{\sigma}_{\alpha}^{L}$ or $\tilde{\sigma}_{\alpha}^{U}, \tilde{k}_{i \alpha}^{L}$ or $\tilde{k}_{i \alpha}^{U}, \tilde{\kappa}_{i \alpha}^{L}$ or $\tilde{\kappa}_{i \alpha}^{U}, i=1,2, \ldots, D$, respectively. From the resolution identity

$$
\mu_{\tilde{C}_{0}}(c)=\sup _{0 \leq \alpha \leq 1} \alpha I_{\left(\tilde{C}_{0}\right)_{\alpha}}(c)
$$

we obtain the membership function of $\tilde{C}_{0}$. The $\alpha$-level sets of $\tilde{C}_{0}$ can be comfortable tools for decision making.

For a given $\alpha$ (e.g., $\alpha=0.95$ ) the $\alpha$-level set of $\tilde{C}_{0}$ can be treated by a financial analyst as the interval of the European call option prices. Then the financial analyst can pick any value from this interval as the option price with an acceptable membership degree. For example, if the market price of the European call option is outside of such an interval, an appropriate course of action (i.e., buying or selling the financial instrument) may be taken by the decision-maker.

\section{Numerical experiments and analysis}

As can be easily seen, the fuzzy pricing formula for the European call option (14) may be calculated numerically. However, for more complicated derivatives, Monte Carlo simulations may be a better approach.

Therefore in this section we present the application of the simulation method for the option pricing in the case of the fuzzy framework. We conduct simulations in a similar manner as in our previous work (Nowak and Romaniuk, 2010). For our analysis we set $D=2$, although simulations may be also applied for other values of $D$.

We fix an $\alpha$-level. For the L-R fuzzy parameters $\tilde{\mu}, \tilde{\sigma}, \tilde{r}, \tilde{k}_{1}, \tilde{k}_{2}, \tilde{\kappa}_{1}, \tilde{\kappa}_{2}$, we find the solution $\theta_{0}$ of (15) using their defuzzified counterparts $\mu^{*}, \sigma^{*}, r^{*}, k_{1}^{*}, k_{2}^{*}, \kappa_{1}^{*}$ and $\kappa_{2}^{*}$. For given $\alpha$-level sets of the parameters we randomly pick the value of each parameter treating the appropriate sets as intervals for uniform distribution. We repeat this procedure $n$ times to find the estimate of $\alpha$-level set $\tilde{C}_{\alpha}$ of the option price. If the closed-form formula for the option price is sufficiently regular (as in the case of (14)), the estimator of $\tilde{C}_{\alpha}$ is given by the minimum and maximum of $n$ samples obtained via the method described above. Apparently, we could find other descriptive statistics apart from the minimum and maximum in the same way. These statistics may be also useful for an experimenter, e.g., if someone is interested in a crisp value of the option price, instead of the whole set $\tilde{C}_{\alpha}$, they may use the median or mean. On the other hand, standard deviation may be treated as an indicator of numerical stability for the simulations.

If the closed formula for the option price is not known, simulations may be applied for an iterative version of process $Y_{t}$, which is analogous to the formula known as the Euler scheme in the Black-Scholes model 
(Glasserman, 2004; Nowak et al., 2002). For example, if $D$ Poisson processes are considered, we have

$$
\begin{aligned}
Y_{t_{i+1}}= & Y_{t_{i}} \exp \left(\left(\mu+\theta_{0} \sigma^{2}\right) \delta t+\sigma \sqrt{\delta t} \epsilon_{i}\right. \\
& \left.+k_{1} \nu_{1 i}+k_{2} \nu_{2 i}+\cdots+k_{D} \nu_{D i}\right),
\end{aligned}
$$

where $i=0,1, \ldots, m$ for $t_{i_{0}}=0$ and $t_{i_{m}}=$ $T, \delta t=t_{i+1}-t_{i}=$ const, $\epsilon_{0}, \ldots \epsilon_{m-1}$ are i.i.d. random variables from the standard normal distribution, $\nu_{10}, \ldots, \nu_{1 m-1}, \nu_{20}, \ldots \nu_{2 m-1}, \ldots, \nu_{D 0}, \ldots, \nu_{2 D-1}$ are i.i.d. realizations from Poisson processes with the intensities $\kappa_{1}^{\prime}, \kappa_{2}^{\prime}, \ldots, \kappa_{D}^{\prime}$, respectively (cf. (12)). The value $m$ is the number of steps in each trajectory.

Then we generate $n$ separate trajectories via the formula (16). For each trajectory, as previously noted, we randomly pick values of the fuzzy parameters. The discounted values for the fixed payment function of the appropriate derivative for $n$ trajectories are used to obtain the estimator for $\tilde{C}_{\alpha}$.

In our numerical experiments we use the formula (14) for a model with two Poisson processes to obtain the estimator of the option price. We assume that $S_{0}=1$ (one monetary unit assumption), $K=0.9$ and expiration date $T=1$ (one year assumption). In each experiment we use $n=10000$ simulations.

6.1. Analysis I. We set $\alpha=0.9$, i.e., the option price will lie in some interval with the membership degree 0.9. The appropriate intervals for the parameters are described in Table 1 As mentioned before, based on the generated samples, we may find descriptive statistics for the option price (see Table 2). Therefore the estimator for $\tilde{C}_{\alpha}$ is given by the interval $[0.109991,0.16266]$ (if the minimum and maximum is used) or by the crisp value 0.136173 (if, e.g., the mean is applied).

Table 1. Parameters given by intervals in Analysis I.

\begin{tabular}{|c|c|}
\hline Parameter & Interval \\
\hline \hline$\tilde{\mu}$ & {$[0.01,0.05]$} \\
$\tilde{r}$ & {$[0.01,0.05]$} \\
$\tilde{\sigma}$ & {$[0.1,0.15]$} \\
$\tilde{\kappa}_{1}$ & {$[0.05,0.1]$} \\
$\tilde{\kappa}_{2}$ & {$[0.05,0.1]$} \\
$\tilde{k}_{1}$ & {$[0.05,0.1]$} \\
$\tilde{k}_{2}$ & {$[-0.1,-0.05]$} \\
\hline
\end{tabular}

6.2. Analysis II. We analyse the option price if some parameters are changed and the others are set as constants. Let us assume that intervals for the parameters $k_{1}>0$ and $k_{2}<0$ are becoming wider, i.e., the value of the right side for the $\tilde{k}_{1}$ interval is getting bigger and the value of the left side for the $\tilde{k}_{2}$ interval is getting lower. In such a way
Table 2. Descriptive statistics for Analysis I.

\begin{tabular}{|c|c|}
\hline Statistics & Value \\
\hline \hline Mean & 0.136173 \\
Standard dev. & 0.0111235 \\
Minimum & 0.109991 \\
$Q_{1}$ & 0.126787 \\
Median & 0.136205 \\
$Q_{3}$ & 0.145481 \\
Maximum & 0.16266 \\
\hline
\end{tabular}

the upward and downward jumps are more important for the trajectory of the underlying asset.

The descriptive statistics for some intervals for the parameters $\tilde{k}_{1}$ and $\tilde{k}_{2}$ may be found in Table 3 Other parameters are the same as described in Table 1 . Some statistics may be also compared in Fig. 1

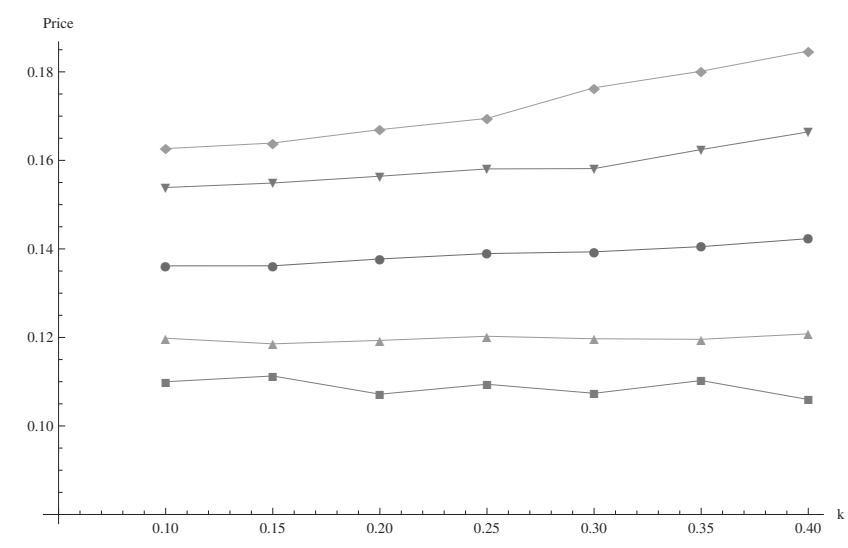

Fig. 1. Graphs for descriptive statistics for option price in Analysis II (rectangles: minimum, triangles: 0.05 quantile, circles: mean, upside down triangles: 0.95 quantile, rhombus: maximum, $\mathrm{X}$ axis: absolute value for the right side of the $k_{1}$ interval and for the left side of the $k_{2}$ interval).

6.3. Analysis III. We analyse the option price as the function of a value $\alpha$. Triangular fuzzy numbers are used to denote the fuzzy parameters $\tilde{\mu}, \tilde{\sigma}, \tilde{r}, \tilde{k}_{1}, \tilde{k}_{2}, \tilde{\kappa}_{1}, \tilde{\kappa}_{2}$ for the case considered (see Table 4). The estimators for the option price for various $\alpha$ are given by the intervals (where the minimum and maximum are used), or by the crisp values (where mean is used) and can be found in Table 5. As we could see, the intervals are becoming wider and crisp values are becoming lower for a lower value of $\alpha$ (see Fig. 2).

6.4. Analysis IV. Next we focus on some statistical properties of the samples generated via the method described earlier. We set $\alpha=0.9$, and the appropriate 
Table 3. Parameters and data for Analysis II.

\begin{tabular}{|l|c|c|}
\hline$\tilde{k}_{1}$ & {$[0.05,0.1]$} & {$[0.05,0.15]$} \\
$\tilde{k}_{2}$ & {$[-0.1,-0.05]$} & {$[-0.15,-0.05]$} \\
\hline \hline Mean & 0.136173 & 0.136181 \\
Standard dev. & 0.0111235 & 0.0111413 \\
Minimum & 0.109991 & 0.111297 \\
$Q_{1}$ & 0.126787 & 0.127886 \\
Median & 0.136205 & 0.135429 \\
$Q_{3}$ & 0.145481 & 0.144312 \\
Maximum & 0.16266 & 0.163922 \\
\hline \hline$\dot{k}_{1}$ & {$[0.05,0.2]$} & {$[0.05,0.25]$} \\
$\tilde{k}_{2}$ & {$[-0.2,-0.05]$} & {$[-0.25,-0.05]$} \\
\hline \hline Mean & 0.137726 & 0.13895 \\
Standard dev. & 0.0114401 & 0.0117028 \\
Minimum & 0.107177 & 0.109422 \\
$Q_{1}$ & 0.128584 & 0.129857 \\
Median & 0.137759 & 0.138936 \\
$Q_{3}$ & 0.146129 & 0.147754 \\
Maximum & 0.166949 & 0.169483 \\
\hline
\end{tabular}

\begin{tabular}{|l|c|c|c|}
\hline$\tilde{k}_{1}$ & {$[0.05,0.3]$} & {$[0.05,0.35]$} & {$[0.05,0.4]$} \\
$\tilde{k}_{2}$ & {$[-0.3,-0.05]$} & {$[-0.35,-0.05]$} & {$[-0.4,-0.05]$} \\
\hline \hline Mean & 0.139338 & 0.140513 & 0.142314 \\
Standard dev. & 0.0120532 & 0.0129048 & 0.0137104 \\
Minimum & 0.10738 & 0.110263 & 0.105973 \\
$Q_{1}$ & 0.130199 & 0.131068 & 0.131789 \\
Median & 0.139361 & 0.140338 & 0.142105 \\
$Q_{3}$ & 0.147899 & 0.149172 & 0.151735 \\
Maximum & 0.176408 & 0.180148 & 0.184716 \\
\hline
\end{tabular}

Table 4. Parameters given by fuzzy numbers in Analysis III.

\begin{tabular}{|c|c|}
\hline Parameter & Triangular fuzzy number \\
\hline \hline$\tilde{\mu}$ & {$[0.02,0.04,0.06]$} \\
$\tilde{r}$ & {$[0.03,0.05,0.7]$} \\
$\tilde{\sigma}$ & {$[0.1,0.2,0.3]$} \\
$\tilde{\kappa}_{1}$ & {$[1,2,4]$} \\
$\tilde{\kappa}_{2}$ & {$[1,2,4]$} \\
$\tilde{k}_{1}$ & {$[0.1,0.2,0.3]$} \\
$\tilde{k}_{2}$ & {$[-0.3,-0.2,-0.1]$} \\
\hline
\end{tabular}

intervals for the parameters can be found in Table 6 The generated samples may be treated as statistical samples and could be easily visualised by common statistical tools, like a histogram (see Fig. 3) or a box-and-whisker plot (see Fig. 4). In our case the samples tend to be right-skewed (see Fig. 3), therefore it may be profitable to use the median as the estimator of the crisp value of the option price instead of the average. However, there are no indications of outliers (see Fig. 4).

Users may be also interested if the samples are distributed according to, e.g., some normal distribution in order to conduct additional statistical tests. Therefore, the Shapiro-Wilk or Kolmogorow-Smirnov tests may be applied. In our case both of these tests reject the
Table 5. Price interval and price crisp value for various $\alpha$ in Analysis III.

\begin{tabular}{|l|c|c|}
\hline$\alpha$ & Price interval & Price crisp value \\
\hline \hline 0.95 & {$[0.214156,0.27526]$} & 0.241641 \\
0.9 & {$[0.181198,0.307302]$} & 0.240797 \\
0.85 & {$[0.158509,0.331152]$} & 0.237491 \\
0.8 & {$[0.143532,0.389082]$} & 0.237128 \\
0.75 & {$[0.121319,0.413456]$} & 0.234365 \\
\hline
\end{tabular}

null hypothesis of normality-p-values are lower than 0.00001 .

\section{Conclusions}

In the paper a geometric Levy process for the underlying asset was introduced. The discontinuous part of the $\log$-price process is a linear combination of independent Poisson processes and it describes positive and negative jumps of the financial instrument prices. The combination of stochastic analysis and fuzzy theory enables us to obtain the pricing formula for the European call option. We prove the valuation formula and presented appropriate simulation techniques for option pricing in the case of the fuzzy framework. 

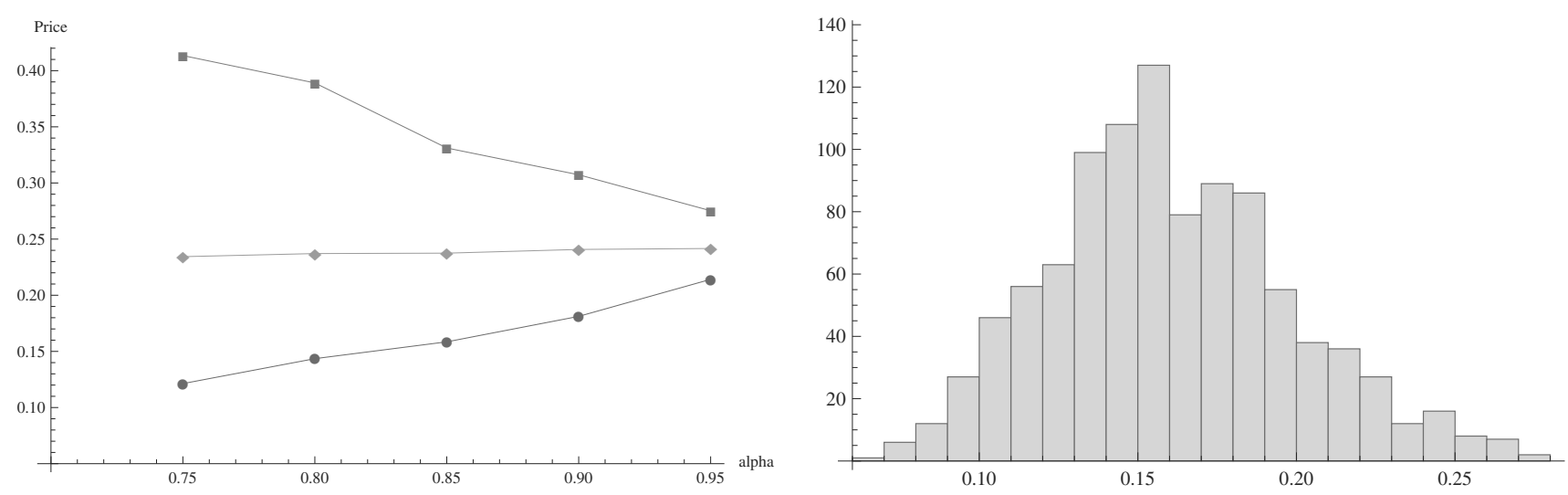

Fig. 2. Graphs for the price intervals (rectangles and circles) and the price crisp values (diamonds) for various $\alpha$ in Analysis III.

Table 6. Parameters given by intervals in Analysis IV.

\begin{tabular}{|c|c|}
\hline Parameter & Interval \\
\hline \hline$\tilde{\mu}$ & {$[0.01,0.05]$} \\
$\tilde{r}$ & {$[0.02,0.07]$} \\
$\tilde{\sigma}$ & {$[0.1,0.15]$} \\
$\tilde{\kappa}_{1}$ & {$[1,2]$} \\
$\tilde{\kappa}_{2}$ & {$[1,2]$} \\
$\tilde{k}_{1}$ & {$[0.05,0.1]$} \\
$\tilde{k}_{2}$ & {$[-0.1,-0.05]$} \\
\hline
\end{tabular}

\section{References}

Bakshi, G., Cao, C. and Chen, Z. (1997). Empirical performance of alternative option pricing models, The Journal of $\mathrm{Fi}$ nance LII(5): 2003-2049.

Bardossy, A. and Duckstein, L. (1995). Fuzzy Rule-Based Modeling with Applications to Geophysical, Biological and Engineering Systems (Systems Engineering), CRC Press, Boca Raton, FL.

Barndorff-Nielsen, O.E. (1998). Processes of normal inverse Gaussian type, Finance and Stochastics 2(1): 41-68.

Bates, D. (1996). Jumps and stochastic volatility: Exchange rate processes implicit in deutschemark options, The Review of Financial Studies 9(1): 69-107.

Black, F. and Scholes, M. (1973). The pricing of options and corporate liabilities, Journal of Political Economy 81(3): 637-659.

Brigo, D., Pallavicini, A. and Torresetti, R. (2007). Credit derivatives: Calibration of $\mathrm{CDO}$ tranches with the dynamical GPL model, Risk Magazine 20(5): 70-75.

Davis, M. (2001). Mathematics of financial markets, in B. Engquist and W. Schmid (Eds.), Mathematics Unlimited-2001 \& Beyond, Springer, Berlin, pp. 361-380.

Dubois, D. and Prade, H. (1980). Fuzzy Sets and SystemsTheory and Applications, Academic Press, New York, NY.

Fig. 3. Histogram based on samples in Analysis IV.

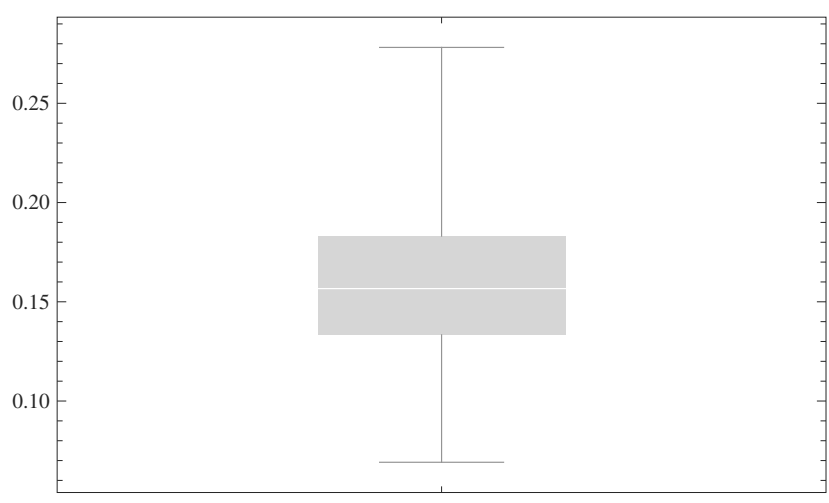

Fig. 4. Box-and-whisker plot based on samples in Analysis IV.

El Karoui, N. and Rouge, R. (2000). Pricing via utility maximization and entropy, Mathematical Finance 10(2): 259-276.

Frittelli, M. (2000). The minimal entropy martingale measure and the valuation problem in incomplete markets, Mathematical Finance 10(1): 39-52.

Fujiwara, T. and Miyahara, Y. (2003). The minimal entropy martingale measures for geometric Levy processes, $\mathrm{Fi}$ nance and Stochastics 7(1): 509-531.

Glasserman, P. (2004). Monte Carlo Methods in Financial Engineering, Springer-Verlag, New York, NY.

Hull, J.C. (1997). Options, Futures and Other Derivatives, Prentice Hall, Upper Saddle River, NJ.

Jacod, J. and Shiryaev, A. (1987). Limit Theorems for Stochastic Processes, Springer-Verlag, Berlin/Heidelberg/New York, NY.

Kou, S.G. (2002). A jump-diffusion model for option pricing, Management Science 48(8): 1086-1101.

Kou, S.G. and Wang, H. (2004). Option pricing under a double exponential jump diffusion model, Management Science 50(9): 1178-1192. 
Li, C. and Chiang, T.-W. (2012). Intelligent financial time series forecasting: A complex neuro-fuzzy approach with multi-swarm intelligence, International Journal of Applied Mathematics and Computer Science 22(4): 787-800, DOI: 10.2478/v10006-012-0058-x.

Madan, D.B. and Seneta, E. (1990). The variance gamma (v.g.) model for share market returns, The Journal of Business 63(4): 511-524.

Merton, R. (1976). Option pricing when underlying stock returns are discontinuous, Journal of Financial Economics 3(1): 125-144.

Miyahara, Y. (2004). A note on Esscher transformed martingale measures for geometric Levy processes, Discussion Papers in Economics, No. 379, Nagoya City University, Nagoya, pp. 1-14.

Nowak, P. (2011). Option pricing with Levy process in a fuzzy framework, in K.T. Atanassov, W. Homenda, O. Hryniewicz, J. Kacprzyk, M. Krawczak, Z. Nahorski, E. Szmidt and S. Zadrożny (Eds.), Recent Advances in Fuzzy Sets, Intuitionistic Fuzzy Sets, Generalized Nets and Related Topics, Polish Academy of Sciences, Warsaw, pp. 155-167.

Nowak, P., Nycz, P. and Romaniuk, M. (2002). On selection of the optimal stochastic model in the option pricing via Monte Carlo methods, in J. Kacprzyk and J. Węglarz (Eds.), Modelling and Optimization-Methods and Applications, Exit, Warsaw, pp. 59-70, (in Polish).

Nowak, P. and Romaniuk, M. (2010). Computing option price for Levy process with fuzzy parameters, European Journal of Operational Research 201(1): 206-210.

Shiryaev, A.N. (1999). Essential of Stochastic Finance, World Scientific Publishing, Singapore.

Ssebugenyi, C.S. (2011). Using the minimal entropy martingale measure to valuate real options in multinomial lattices, $A p$ plied Mathematical Sciences 67(5): 3319-3334.

Wu, H.-C. (2004). Pricing European options based on the fuzzy pattern of Black-Scholes formula, Computers \& Operations Research 31(7): 1069-1081.

Wu, H.-C. (2007). Using fuzzy sets theory and Black-Scholes formula to generate pricing boundaries of European options, Applied Mathematics and Computation 185(1): 136-146.
Xu, W.D., Wu, C.F. and Li, H.Y. (2011). Foreign equity option pricing under stochastic volatility model with double jumps, Economic Modeling 28(4): 1857-1863.

Yoshida, Y. (2003). The valuation of European options in uncertain environment, European Journal of Operational Research 145(1): 221-229.

Zadeh, L.A. (1965). Fuzzy sets, Information and Control 8(47): 338-353.

Zhang, L.-H., Zhang, W.-G., Xu, W.-J. and Xiao, W.-L. (2012) The double exponential jump diffusion model for pricing European options under fuzzy environments, Economic Modelling 29(3): 780-786.

Zhou, C. (2002). Fuzzy-arithmetic-based Lyapunov synthesis in the design of stable fuzzy controllers: A computing-with-words approach, International Journal of Applied Mathematics and Computer Science 12(3): 411-421.

Piotr Nowak received his M.Sc. degree in theoretical mathematics from the Faculty of Mathematics, Informatics and Mechanics, University of Warsaw, Poland. After completing doctoral studies, he received his Ph.D. degree in mathematics from the University of Warsaw in 1999. The main topics of his current research at the Systems Research Institute of the Polish Academy of Sciences are stochastic analysis, financial mathematics, probability and statistics.

Maciej Romaniuk received his M.Sc. degree in applied mathematics from the Faculty of Mathematics, Informatics and Mechanics, University of Warsaw, Poland. After completing doctoral studies, he received his Ph.D. degree in informatics from the Systems Research Institute of the Polish Academy of Sciences in 2007. The main topics of his current research at the Systems Research Institute are simulations, financial mathematics and statistics.
Received: 17 July 2012

Revised: 24 January 2013 